MORE IS BETTER

\title{
More (of the Right Strategies) is Better: Disaggregating the Naturalistic Between- and Within-Person Structure and Effects of Emotion Regulation Strategies
}

\author{
Matthew W. Southward1 \& Jennifer S. Cheavens2 \\ 1Department of Psychology, University of Kentucky, USA \\ ${ }_{2}$ Department of Psychology, The Ohio State University, USA
}

\begin{abstract}
Author Note
Matthew W. Southward: https://orcid.org/0000-0002-5888-2769

Jennifer S. Cheavens: https://orcid.org/0000-0002-3778-9346

We have no known conflicts of interest to disclose.

Correspondence concerning this article should be addressed to Matthew W. Southward,
\end{abstract} University of Kentucky, 343 Waller Ave., Suite 303, Lexington, KY 40504. Phone: 859-562-

1569. Email: southward@uky.edu

We would like to thank Jane E. Heiy for her efforts in the original study design and data collection.

In press at Cognition \& Emotion

https://doi.org/10.1080/02699931.2020.1797637 


\begin{abstract}
Although people often use multiple strategies to regulate their emotions, it is unclear if using more strategies effectively changes emotional outcomes. This may be because there is no clear, data-driven structure to organise which strategies people use together, so strategies with opposing impacts are modelled together. We first conducted a multilevel factor analysis of negative- and positive-emotion regulation strategies among undergraduates $(n=92)$ completing ecological momentary assessment three times per day for 10 days. Solutions including 3within/3-between factors were most interpretable. Using more between-person Adaptive Engagement strategies and within-person Adaptive Engagement, Enhancement, and Behavioural strategies predicted improved mood, whereas using more between-person Aversive Cognitive and within-person Aversive Cognitive and Disengagement strategies predicted worse mood, $p$ s < .05 . Using a greater quantity of strategies may thus promote better, or worse, emotional outcomes, depending on the class of strategies used.

Keywords: emotion regulation, flexibility, ecological momentary assessment, affect, HLM
\end{abstract}




\section{Theories of Emotion Regulation Strategy Use}

Emotion regulation is the process of altering the onset, intensity, and/or duration of emotions (Gross \& Thompson, 2007) and can be accomplished in many ways. In fact, undergraduate students used seven emotion regulation strategies on average in individual emotional experiences (Heiy \& Cheavens, 2014). Researchers have developed two prominent theories to characterise the effects of multiple emotion regulation strategies. First, Bonanno and Burton's (2013) theory of regulatory flexibility posits that people with larger repertoires of emotion regulation strategies, defined as the number of strategies a person can access, tend to report better emotional health. In this between-person theory, people with larger repertoires are thought to have more options to regulate their emotions, so they can better adapt to changing environmental demands. Larger repertoires are also thought to be more beneficial regardless of the strategies they comprise. The authors argue specific strategies cannot be defined as putatively adaptive (i.e., generally promoting beneficial emotional outcomes) or maladaptive (i.e., generally promoting detrimental emotional outcomes) because (mal)adaptiveness depends on contextual factors, although others have disputed this claim (Aldao et al., 2010).

Second, Ford et al.'s (2019) theory of emotion polyregulation characterizes the withinperson process of using multiple strategies in specific emotional experiences. The authors propose that using more "synergistic" adaptive emotion regulation strategies together (e.g., distraction followed by reappraisal) may promote more effective emotional changes than simply using a larger number of strategies, because "synergistic" adaptive strategies effectively complement each other. In their example, distraction offers quick relief while reappraisal facilitates better long-term emotional processing. On the other hand, multiple maladaptive strategies may "synergistically" increase negative emotions by generating new stressors (i.e., 
rumination may intensify negative memories, prompting the use of self-harm and new negative consequences).

\section{Are More Strategies Better? Empirical Findings}

Researchers have reported mixed findings regarding the effectiveness of more strategies. Between-persons, undergraduates higher in conscientiousness and lower in borderline personality disorder features reported larger repertoires of strategies (Southward et al., 2018), whereas people who reported more intense negative affect when watching a disgust-eliciting video used more strategies (Aldao \& Nolen-Hoeksema, 2013). Within-persons, using more strategies to regulate negative emotions was unrelated to mood but using more strategies to regulate positive emotions was related to better mood (Heiy \& Cheavens, 2014). These mixed findings may result from a lack of meaningful structure among strategies because strategies that down-regulate negative emotions and strategies that up-regulate negative emotions, for instance, are included together.

\section{The Empirical Structure of Emotion Regulation}

Researchers have conducted two ecological momentary assessment (EMA) studies using multilevel exploratory factor analyses (EFAs) to assess the structure of regulatory strategies. Multilevel EFAs can measure emotion regulation strategies at the between-person (i.e., which strategies tend to be used together on average by different people) and within-person (i.e., which strategies tend to be used together over time by any given person) levels. Roesch et al. (2010) assessed 14 coping strategies among undergraduates. They found four between- and withinperson factors labelled cognitive/avoidant, problem-solving, social support, and emotional expression. Greater within-person cognitive/avoidant and problem-solving use was associated with greater positive affect but unrelated to negative affect, whereas within-person emotional 
expression was associated with greater negative and lower positive affect.

McMahon and Naragon-Gainey (2019) assessed eleven strategies among undergraduate and treatment-seeking participants. They found two between-person factors: Avoidance and Engagement. Among undergraduates, four within-person factors including Attentional Shift, Acceptance, Avoidance, and Emotional Expression best fit the data. Among treatment-seeking participants, three within-person factors (Attentional Shift, Avoidance, Emotional Expression) fit best. Although within-person Avoidance was associated with greater negative affect and lower positive affect, within-person Attentional Shift was associated with greater negative affect and lower positive affect among undergraduates. Within-person Acceptance was also only associated with greater positive affect among undergraduates.

Naragon-Gainey et al. (2017) further explored the between-person structure of emotion regulation strategies in a meta-analytic factor analysis. A three-factor solution best characterised the habitual use of ten emotion regulation strategies: Adaptive Engagement (problem-solving, cognitive reappraisal, mindfulness), Aversive Cognitive Perseveration (worry, rumination, experiential avoidance), and Disengagement (distraction, behavioural avoidance, expressive suppression).

Together, these results suggest that emotion regulation strategies exhibit a two- to fourfactor structure between- and within-persons. Differences in factor structures may have resulted from the relatively limited number of strategies examined. Further, each study modelled latent factors of emotion regulation strategies, which give greater weight to higher-loading strategies, obscuring whether (a) using a greater number of strategies or (b) using one or two higher-loading strategies is related to emotional outcomes. Finally, these studies included only one or two strategies that up-regulate positive emotions. Given the role of positive emotions in healthy 
psychological functioning (Fredrickson, 2004), it is necessary to understand how people effectively enhance positive emotions.

\section{Current Study}

To address differences among previous studies regarding the structure of emotion regulation strategies, we conducted a secondary data analysis of Heiy and Cheavens (2014) using multilevel EFAs of a larger selection of strategies for both negative $(N=20)$ and positive $(N=$ 20) emotions. We determined the most appropriate factor structures based on theoretical considerations and empirical results. We hypothesized (1) that two-to-four emotion regulation factors would best fit the data. Our primary question of interest concerned Bonnano and Burton's (2013) and Ford et al.'s (2019) theories that using more strategies, between- and within-persons, would promote better emotional functioning. We tested whether strategies from each factor predicted changes in mood using disaggregated between- and within-person strategy counts. We hypothesized (2) that using greater numbers of putatively adaptive strategies in general and in the moment would predict improved mood whereas using greater numbers of putatively maladaptive strategies would predict worse mood in response to negative and positive emotions.

\section{Materials and Methods}

\section{Participants}

Participants were undergraduates $(n=92)$ from a large Midwestern university enrolled in introductory psychology classes who received class credit. Participants were 19.73 years old (SD $=2.25)$ on average. Most identified as female (54\%) and Caucasian (81\%). Thirty-two participants were invited based on elevated neuroticism scores at pre-screening 1 , resulting in a relatively normal distribution on this construct. See Heiy and Cheavens (2014) for a complete

\footnotetext{
1 Based on the NEO Personality Inventory-Revised, Neuroticism subscale (Costa \& McCrae, 1992). Items from this
} measure demonstrated excellent internal consistency in the current sample (Cronbach's $\alpha=.95)$. 
description of demographic and personality variables.

\section{Study Procedure}

Participants were recruited through a university psychology student study pool. All participants provided informed consent, completed baseline measures, and received instruction in study procedures at an in-person session. Participants completed all EMA prompts on a Palm PilotTm z22 personal device assistant. At the start of each prompt, participants rated their mood on a visual analogue scale from 0 (worst mood) to 100 (best mood). Participants then reported if they had experienced a negative and/or positive emotion since the previous prompt. If participants reported a negative emotion, they then reported every negative-emotion regulation strategy used to lessen the intensity of that negative emotion from 20 options (Table S1). If participants reported a positive emotion, they then reported every positive-emotion regulation strategy used to maintain or increase the intensity of that positive emotion from 20 options (Table S2). These 40 strategies were selected based on commonly used measures of habitual emotion regulation to extend research on habitual emotion regulation to patterns of momentary emotion regulation (Tables S1-S2). Participants were prompted for responses three times per day for ten days at random times within 4-hr windows during anticipated waking hours.

\section{Data Analytic Method}

We conducted multilevel EFAs using Mplus 7.0 (Muthén \& Muthén, 2012) to disaggregate between- and within-person variability. We conducted separate analyses among negative- and positive-emotion regulation strategies, assessing up to four latent factors at each level using maximum likelihood estimation with robust standard errors. We used oblique geomin rotations to test correlations among the resulting factors. To determine the most appropriate solution, we relied on factor interpretability and fit indices: root-mean-square error of 
approximation $(\mathrm{RMSEA} \leq .06)$, comparative fit index $(\mathrm{CFI} \geq .95)$, Tucker-Lewis Index (TLI $\geq$ .95), and standardised root-mean-square residual (SRMR $\leq .08 ; \mathrm{Hu} \&$ Bentler, 1999).

We then grouped observed emotion regulation variables by their relevant factors.2 We calculated between-person scores by taking the average number of strategies from each group each participant used across all occasions and grand-mean-centering these scores. We calculated within-person scores by taking the difference between the number of strategies used in a given occasion and each person's average number of strategies used from that group across all occasions.

We examined between- and within-person means, standard deviations, and correlations. Because we conducted four mean comparisons, we used a Bonferroni-corrected $\alpha$ of $.05 / 4=$ .0125. We calculated within-person correlations using the rmcorr package (Version 0.3.0) in $\mathrm{R}$ (Version 3.6.1), which calculates an average within-person regression slope using repeated measures ANCOVA and computes the correlation coefficient based on the ratio of sums of squares (Bakdash \& Marusich, 2017).

We then used hierarchical linear modelling (HLM) to test the effects of emotion regulation strategies on affective outcomes using proc mixed in SAS (Version 9.4). We regressed mood at the current occasion on the between- and within-person sums of strategies used since the previous occasion from each group using restricted maximum likelihood estimation with random intercepts of participants and emotion type nested within participants, an autoregressive lag-1 residual covariance structure, and the Kenward-Roger method for calculating degrees of freedom. We included mood at the previous occasion and time since the previous occasion to

2 We used observed variables to enhance the interpretability of our results (i.e., a one-unit increase in emotion regulation indicates the use of one more emotion regulation strategy), recognizing that such scoring implies a different factor structure (i.e., loadings fixed to 1) than fit by our EFAs. 
model autoregressive and temporal effects, respectively (Wang \& Maxwell, 2015), and we included emotion type as a covariate to account for experiences of multiple emotions. We used the $r 2 M L M$ function (Rights \& Sterba, 2019) in $\mathrm{R}$ to calculate pseudo- $R 2$. All code is available at https://osf.io/g7b5p/?view_only=31e6c8e4ccad4c62a90fdd64e748beea.

\section{Results}

\section{Negative-Emotion Regulation \\ Exploratory Factor Analyses}

The majority of variability in negative-emotion regulation strategies was within-person (ICCs < .50) for all but two strategies (i.e., substance use, exercise; Table S3). We compared model fit indices across between- and within-person levels and number of factors (Table S4). Due to the low frequency of NSSI, models with four within-person factors did not converge. We re-ran our EFAs without NSSI; these models converged for all solutions. The best-fitting model of the solutions with NSSI removed included 4-within/4-between factors, which demonstrated acceptable-to-excellent fit across indices (Table S5). However, the interpretability of this structure was less evident than the 3-within/3-between solution with NSSI included. For instance, in the 4-within/4-between solution without NSSI, one within-person factor included positive refocusing and social support in addition to other-blame, sleep, and exercise, and one between-person factor only included two strategies: sleep and exercise (Table S6). These factors are arguably less interpretable than the 3-within/3-between factor solution with NSSI, which demonstrated excellent absolute fit (RMSEA $=.019,90 \%$ CI $[.012, .025])$, better aligns with previous meta-analytic work (Naragon-Gainey et al., 2017) and includes NSSI, a clinically meaningful variable. Although we focused on strategy categories from the 3-within/3-between solution, we include results using the 4-within/4-between solution in supplemental materials. 
Using Naragon-Gainey et al.’s (2017) factor labels, the 3-within/3-between solution consisted of Adaptive Engagement, Aversive Cognitive Perseveration, and Disengagement strategies (Table 1), which were relatively independent. The specific strategies associated with each factor differed somewhat within- and between-persons (Table 1).

\section{Negative-Emotion Regulation Strategy Use}

Between-person. Averaging the number of strategies used across the study, participants used greater numbers of Adaptive Engagement strategies $(M=3.32, S D=1.38)$ than both Aversive Cognitive $(M=2.68, S D=1.25), t(91)=3.98, p<.01,95 \%$ CI $[.33, .97]$, and Disengagement $(M=.99, S D=.67), t(91)=16.44, p<.01,95 \%$ CI $[2.05,2.62]$, strategies (Table S8). Participants also used greater numbers of Aversive Cognitive than Disengagement strategies, $t(91)=12.37, p<.01,95 \%$ CI $[1.42,1.96]$. Adaptive Engagement strategies were correlated with Aversive Cognitive, $r=.30, p<.01$, and Disengagement strategies, $r=.27, p<$ .01 , but Aversive Cognitive strategies were not significantly related to Disengagement strategies, $r=.17, p=.10$. Only people who used greater numbers of Aversive Cognitive strategies tended to be higher in neuroticism, $r=.33, p<.01$.

Within-person. Within-person Adaptive Engagement strategy use was associated with more Aversive Cognitive strategies, $r=.13, p<.01$. However, Adaptive Engagement and Aversive Cognitive strategies were unrelated to Disengagement strategies, $r s<.06, p s>.15$.

\section{Negative-Emotion Regulation Strategies Predicting Change in Mood}

Greater numbers of between-, $B=2.60, S E=1.07, p=.02,95 \%$ CI $[.47,4.73]$, and within-person, $B=1.67, S E=.38, p<.01,95 \%$ CI [.93, 2.42], Adaptive Engagement strategy use predicted improved mood, whereas greater numbers of between-, $B=-2.54, S E=1.19, p=$ $.04,95 \%$ CI $[-4.92,-.17]$, and within-person, $B=-1.77, S E=.56, p<.01,95 \%$ CI $[-2.88,-.66]$, 
Aversive Cognitive strategy use predicted worse mood (Table S9).3 Greater numbers of withinperson, but not between-person, Disengagement strategy use predicted worse mood, $B=-1.27$, $S E=.60, p=.04,95 \% \mathrm{CI}[-2.45,-.09]$. Between-person strategy use explained more variability in mood (pseudo- $R_{2}=.09$ ) than within-person strategy use (pseudo- $R_{2}=.05$ ).

\section{Positive-Emotion Regulation}

\section{Exploratory Factor Analyses}

The majority of variability in positive-emotion regulation strategies was within-person for all but one strategy (substance use; Table S3). The best-fitting model of positive-emotion regulation strategies was a 4-within/4-between solution (Table S4). However, this solution was less interpretable than the 3-within/3-between solution. Specifically, the 3-within/3-between solution included Enhancement, Disengagement, and Behavioural factors (Table 2). Items in the 4-within/4-between solution (Table S7) loaded similarly onto the Disengagement and Behavioural factors but split the Enhancement factor. The two within-person factors resulting from this factor split were (A) consequences, self-credit, replaying, and capitalizing, and (B) savouring, broadening, reminiscing, future focus, social sharing, other-credit, and stimulus control. It was not clear that this division was as meaningful as the 3-within/3-between solution which also demonstrated acceptable-to-excellent fit across most indices. Although we focused on strategy categories from the 3-within/3-between solution, we include results using the 4within/4-between solution in supplemental materials.

\section{Positive-Emotion Regulation Strategy Use}

Between-person. Participants used greater numbers of Enhancement $(M=5.05, S D=$ 2.19) than Disengagement $(M=.67, S D=.81), t(90)=20.65, p<.01,95 \%$ CI [3.95, 4.79], and 
Behavioural strategies $(M=1.59, S D=.80), t(90)=17.13, p<.01,95 \%$ CI $[3.05,3.85]$ (Table S7). Participants also used greater numbers of Behavioural than Disengagement strategies, $t(90)$ $=10.06, p<.01,95 \%$ CI $[.74,1.11]$. Participants who used greater numbers of strategies from any group to regulate positive emotions also used greater numbers of strategies from the other groups, $r \mathrm{~s}: .39-.50, p \mathrm{~s}<.01$. Strategy use was not significantly related to mood or neuroticism, $p s>.10$.

Within-person. Within-person Enhancement, $r=.19, p<.01$, and Behavioural, $r=.13, p$ $<.01$, strategy use was associated with better mood. Enhancement, Behavioural, and Disengagement strategies exhibited small but positive correlations, $r \mathrm{~s}: .10-.14$, $p \mathrm{~s}<.01$.

\section{Positive-Emotion Regulation Strategies Predicting Change in Mood}

Greater numbers of within-person Enhancement, $B=1.03, S E=.24, p<.01,95 \% \mathrm{CI}$ $[.55,1.51]$, and Behavioural strategies, $B=1.26, S E=.50, p<.01,95 \%$ CI $[.27,2.24]$, predicted improved mood (Table S9).4 However, between-person Enhancement and Behavioural strategy use was unrelated to mood, $p \mathrm{~s}>.50$. Within- and between-person Disengagement strategy use was unrelated to mood, $p \mathrm{~s}>.10$. Between-person strategy use explained less variability in mood (pseudo- $\left.R_{2}=.01\right)$ than within-person strategy use (pseudo- $R_{2}=.03$ ).

\section{Discussion}

We explored the multilevel factor structure of negative- and positive-emotion regulation strategies. In line with hypothesis (1), negative-emotion regulation strategies loaded onto three between- and within-person factors: Adaptive Engagement, Aversive Cognitive, and Disengagement. These factors map well onto Naragon-Gainey et al.'s (2017) meta-analytic emotion regulation factors and clarify the factors obtained by McMahon and Naragon-Gainey

4 For full results with the four-factor solution, see Table S10. 
(2019) and Roesch et al. (2010). In line with hypothesis (2), using more Adaptive Engagement strategies habitually and momentarily predicted improvements in mood, whereas using more Aversive Cognitive strategies habitually and momentarily and using more Disengagement strategies momentarily predicted worse mood. These results provide the first direct evidence that the use of more negative-emotion regulation strategies from certain categories predicts improvements in or worsening of mood. These results also suggest that Heiy and Cheavens' (2014) null results regarding the association between total negative-emotion regulation strategy use and mood were likely due to a lack of meaningful emotion regulation structure. Our results support Ford et al.'s (2019) theory of emotion polyregulation regarding the effects of multiple "synergistic" adaptive strategies (i.e., Adaptive Engagement) and extend this theory to the effects of multiple "synergistic" maladaptive strategies (i.e., Aversive Cognitive and Disengagement).

We also examined positive-emotion regulation strategies. In line with hypothesis (1), these strategies were characterised as Enhancement, Disengagement, or Behavioural. In line with hypothesis (2), using more Enhancement and Behavioural strategies momentarily predicted improved mood. These results provide the first direct evidence that the use of more positiveemotion regulation strategies, specifically Enhancement and Behavioural strategies, predicts improvements in mood. This novel finding supports Fredrickson's (2004) broaden-and-build theory in which positive emotions prompt the use of a broader repertoire of thoughts and behaviours. The use of more Enhancement and Behavioural strategies may function as a positive feedback loop to further increase positive emotion intensity. Although the within-person use of more Disengagement strategies was not significantly related to decreases in mood, the size of the effect was comparable to that of Enhancement and Behavioural strategies. Because Disengagement strategies were used least frequently of all three categories (Table S3), this may 
have reduced our power to detect a significant effect. It is possible that people used Disengagement strategies in circumscribed situations (e.g., achievement-oriented social situations; Kalokerinos et al., 2014).

This study is not without limitations. Given cross-cultural (Ford \& Mauss, 2015) and agerelated (Eldesouky \& English, 2018) differences in emotion regulation, our results may not generalise beyond American young adults. Although we assessed participants multiple times each day and explicitly modelled time and previous mood in our analyses, we were unable to assess the temporal unfolding of strategies (Guiller et al., 2019). That is, our design precluded us from capturing the intensity of emotions experienced immediately preceding regulation between timepoints. Thus, our results should be interpreted as a conservative estimate of the naturalistic relation between emotion regulation strategies and momentary changes in emotion intensity. Finally, although we assessed a wide range of strategies, we included relatively few interpersonal emotion regulation strategies (e.g., social support).

Overall, our findings replicate the factor structure of emotion regulation strategies used in response to negative emotions and extend it to positive-emotion regulation. By disaggregating within- from between-person effects, we demonstrated how people use strategies in a given situation and differ in their habitual use. Although the between- and within-person factor structures were largely similar among negative- and positive-emotion regulation strategies, the differing effects on mood among positive-emotion regulation strategies shows the importance of disaggregation. By modelling the number of strategies in a given situation, our results explicitly characterise the effects of using a greater quantity of strategies. Together, these results provide empirical evidence for Ford et al.'s (2019) theory of emotion polyregulation, suggesting both which and how many strategies a person uses influence their emotional dynamics. 


\section{References}

Aldao, A., Nolen-Hoeksema, S., \& Schweizer, S. (2010). Emotion-regulation strategies across psychopathology: A meta-analytic review. Clinical Psychology Review, 30, 217-237. https://doi.org/10.1016/j.cpr.2009.11.004

Aldao, A., \& Nolen-Hoeksema, S. (2013). One versus many: Capturing the use of multiple emotion regulation strategies in response to an emotion-eliciting stimulus. Cognition and Emotion, 27(4), 753-760. https://doi.org/10.1080/02699931.2012.739998

Bakdash, J.Z., \& Marusich, L.R. (2017). Repeated measures correlation. Frontiers in Psychology, 8, 456. https://doi.org/10.3389/fpsyg.2017.00456

Bonanno, G.A., \& Burton, C.L. (2013). Regulatory flexibility: An individual difference perspective on coping and emotion regulation. Perspectives on Psychological Science, 8(6), 591-612. https://doi.org/10.1177/1745691613504116

Cheng, C., Lau, H.-P.B., \& Chan, M.-P.S. (2014). Coping flexibility and psychological adjustment to stressful life changes: A meta-analytic review. Psychological Bulletin, 140(6), 1582-1607. https://doi.org/10.1037/a0037913

Costa, P.T., \& McCrae, R.R. (1992). Revised NEO Personality Inventory (NEO-PI-R) and NEO Five-Factor Inventory (NEO-FFI) professional manual. Psychological Assessment Resources.

Eldesouky, L., \& English, T. (2018). Another year older, another year wiser? Emotion regulation strategy selection and flexibility across adulthood. Psychology and Aging, 33(4), 572585. https://doi.org/10.1037/pag0000251

Ford, B.Q., Gross, J.J., \& Gruber, J. (2019). Broadening our field of view: The role of emotion polyregulation. Emotion Review, 11(3), 197-208. 
https://doi.org/10.1177/1754073919850314

Ford, B.Q., \& Mauss, I.B. (2015). Culture and emotion regulation. Current Opinion in Psychology, 3, 1-5. https://doi.org/10.1016/j.copsyc.2014.12.004

Fredrickson, B.L. (2004). The broaden-and-build theory of positive emotions. Philosophical Transactions of the Royal Society B-Biological Sciences, 359(1449), 1367-1377. https://doi.org/10.1098/rstb.2004.1512

Guiller, T., Dauvier, B., Pavani, J.-B., Chakri, K.H., \& Congard, A. (2019). 'It might be time to accept'. Exploring the dynamics between affect regulation strategies, anxiety and timing of regulation. Personality and Individual Differences, 142, 21-27. https://doi.org/10.1016/j.paid.2019.01.023

Heiy, J.E., \& Cheavens, J.S. (2014). Back to basics: A naturalistic assessment of the experience and regulation of emotion. Emotion, 14(5), 878-891. https://doi.org/10.1037/a0037231

Hu, L., \& Bentler, P.M. (1999). Cutoff criteria for fit indexes in covariance structure analysis: Conventional criteria versus new alternatives. Structural Equation Modeling, 6(1), 1-55. https://doi.org/10.1080/10705519909540118

Kalokerinos, E.K., Greenaway, K.H., Pedder, D.J., \& Margetts, E.A. (2014). Don’t grin when you win: The social costs of positive emotion expression in performance situations. Emotion, 14(1), 180-186. https://doi.org/10.1037/a0034442

McMahon, T.P., \& Naragon-Gainey, K. (2018). The moderating effect of maladaptive emotion regulation strategies on reappraisal: A daily diary study. Cognitive Therapy and Research, 42, 556-564. https://doi.org/10.1007/s10608-018-9913-x

McMahon, T.P., \& Naragon-Gainey, K. (2019). The multilevel structure of daily emotionregulation-strategy use: An examination of within- and between-person associations in 
naturalistic settings. Clinical Psychological Science, 7(2), 321-339.

https://doi.org/10.1177/2167702618807408

Muthén, L.K., \& Muthén, B.O. (1998-2012). Mplus user's guide (7th ed.). Los Angeles, CA: Muthén \& Muthén.

Naragon-Gainey, K., McMahon, T.P., \& Chacko, T.P. (2017). The structure of common emotion regulation strategies: A meta-analytic examination. Psychological Bulletin, 143(4), 384427. https://doi.org/10.1037/bu10000093

Pinheiro, J., Bates, D., DebRoy, S., Sarkar, D., \& R Core Team. (2019). nlme: Linear and nonlinear mixed effects models (Version 3.1-141). Retrieved from https://CRAN.Rproject.org/package $=$ nlme

Rights, J.D., \& Sterba, S.K. (2019). Quantifying explained variance in multilevel models: An integrative framework for defining R-squared measures. Psychological Methods, 24(3), 309-338. https://doi.org/10.1037/met0000184

Roesch, S.C., Aldridge, A.A., Stocking, S.N., Villodas, F., Leung, Q., Bartley, C.E., \& Black, L.J. (2010). Multilevel factor analysis and structural equation modeling of daily diary coping data: Modeling trait and state variation. Multivariate Behavioral Research, 45(5), 767-789. https://doi.org/10.1080/00273171.2010.519276

Southward, M.W., Altenburger, E.A., Moss, S.A., Cregg, D.R., \& Cheavens, J.S. (2018). Flexible, yet firm: A model of healthy emotion regulation. Journal of Social and Clinical Psychology, 37(4), 231-251. https://doi.org/10.1521/jscp.2018.37.4.231

Wang, L., \& Maxwell, S.E. (2015). On disaggregating between-person and within-person effects with longitudinal data using multilevel models. Psychological Methods, 20(1), 63-83. https://doi.org/10.1037/met0000030 


\section{Table 1}

Negative-Emotion Regulation Factor Loadings

Within-person

\begin{tabular}{|c|c|c|c|}
\hline Variable & $\begin{array}{c}\text { Adaptive } \\
\text { Engagement }\end{array}$ & $\begin{array}{c}\text { Aversive } \\
\text { Cognitive } \\
\text { Perseveration }\end{array}$ & Disengagement \\
\hline Acceptance & $.69 *(.08)$ & $-.10(.13)$ & $.01(.05)$ \\
\hline Benefit finding & $.61 *(.06)$ & $.27 *(.12)$ & $.00(.03)$ \\
\hline Perspective & $.52 *(.07)$ & $.02(.10)$ & $.08(.09)$ \\
\hline Problem-solving & $.41 *(.07)$ & $.33^{*}(.09)$ & $-.04(.11)$ \\
\hline Reappraisal & $.34 *(.08)$ & $.09(.14)$ & $.31 *(.09)$ \\
\hline Behavioral activation & $.27 *(.06)$ & $-.07(.09)$ & $.06(.09)$ \\
\hline Positive refocusing & $.26 *(.08)$ & $-.23 *(.11)$ & $.14(.14)$ \\
\hline Exercise & $.23 *(.10)$ & $-.02(.09)$ & $.25^{*}(.11)$ \\
\hline Social support & $.15(.08)$ & $.05(.08)$ & $-.02(.09)$ \\
\hline Consequences & $.04(.09)$ & $.62 *(.10)$ & $.33(.24)$ \\
\hline Rumination & $.01(.05)$ & $.54 *(.06)$ & $.13(.24)$ \\
\hline Generalizing & $-.02(.04)$ & $.49 *(.15)$ & $.51 *(.19)$ \\
\hline Self-blame & $.14(.08)$ & $.42 *(.08)$ & $.10(.19)$ \\
\hline NSSI & $-.46^{*}(.18)$ & $.02(.03)$ & $.60 *(.19)$ \\
\hline Substance use & $-.22(.12)$ & $-.08(.16)$ & $.59 *(.10)$ \\
\hline Denial & $.01(.05)$ & $-.46^{*}(.16)$ & $.48 *(.21)$ \\
\hline Expressive suppression & $.13(.08)$ & $-.21(.14)$ & $.38 *(.14)$ \\
\hline Emotional suppression & $.00(.06)$ & $-.36^{*}(.14)$ & $.38 *(.17)$ \\
\hline Other-blame & $-.15 *(.07)$ & $-.03(.09)$ & $.15(.08)$ \\
\hline Sleep & $-.11(.09)$ & $.08(.08)$ & $.14(.09)$ \\
\hline \multicolumn{4}{|l|}{ Correlations } \\
\hline \multicolumn{4}{|l|}{ Adaptive Engagement } \\
\hline Aversive Cognitive & -.02 & & \\
\hline Disengagement & $.25^{*}$ & -.01 & \\
\hline
\end{tabular}

Between-person

\begin{tabular}{|c|c|c|c|}
\hline Variable & $\begin{array}{c}\text { Adaptive } \\
\text { Engagement }\end{array}$ & $\begin{array}{c}\text { Aversive } \\
\text { Cognitive/ } \\
\text { Behavioural }\end{array}$ & Disengagemen \\
\hline Acceptance & $.85 *(.09)$ & $-.35(.25)$ & $-.03(.10)$ \\
\hline Positive refocusing & $.85 *(.11)$ & $-.14(.24)$ & $.16(.22)$ \\
\hline Perspective & $.81 *(.10)$ & $-.11(.25)$ & $-.01(.06)$ \\
\hline Benefit finding & $.73 *(.11)$ & $.17(.22)$ & $-.33(.18)$ \\
\hline Reappraisal & $.70 *(.12)$ & $.08(.22)$ & $.09(.16)$ \\
\hline Problem-solving & $.68 *(.11)$ & $-.26(.21)$ & $.06(.17)$ \\
\hline Behavioral activation & $.58 *(.12)$ & $-.00(.06)$ & $.23(.19)$ \\
\hline Exercise & $.37 *(.17)$ & $.07(.18)$ & $.30(.17)$ \\
\hline Consequences & $-.02(.11)$ & $1.01 *(.06)$ & $-.03(.10)$ \\
\hline Generalizing & $.11(.11)$ & $.91 *(.07)$ & $.03(.08)$ \\
\hline Rumination & $.10(.11)$ & $.83 *(.10)$ & $-.07(.12)$ \\
\hline Other-blame & $-.01(.07)$ & $.60 *(.10)$ & $-.20(.14)$ \\
\hline Substance use & $.24(.12)$ & $.58 *(.12)$ & $.02(.10)$ \\
\hline Self-blame & $-.02(.12)$ & $.44 *(.12)$ & $.35 *(.14)$ \\
\hline Social support & $.13(.14)$ & $.26 *(.13)$ & $-.09(.15)$ \\
\hline Denial & $.00(.07)$ & $-.05(.12)$ & $.94 *(.12)$ \\
\hline Emotional suppression & $-.14(.15)$ & $.00(.02)$ & $.81 *(.10)$ \\
\hline NSSI & $.05(.24)$ & $.29(.21)$ & $.64 *(.22)$ \\
\hline Sleep & $.14(.15)$ & $.04(.13)$ & $.44 *(.15)$ \\
\hline Expressive suppression & $-.03(.11)$ & $.28 *(.11)$ & $.38 *(.15)$ \\
\hline \multicolumn{4}{|l|}{ Correlations } \\
\hline \multicolumn{4}{|l|}{ Adaptive Engagement } \\
\hline Aversive Cognitive & .19 & & \\
\hline Disengagement & $.23^{*}$ & .05 & \\
\hline
\end{tabular}


Note. NSSI = Non-suicidal self-injury. Bold loadings indicate the factor with which the item was grouped. Values in parentheses are standard errors of factor loadings. $* p<.05$ 
Table 2

Positive-Emotion Regulation Factor Loadings

Within-person

\begin{tabular}{|c|c|c|c|}
\hline Variable & Enhancement & Disengagement & Behavioural \\
\hline Savouring & $.67 *(.07)$ & $-.25(.16)$ & $.13(.11)$ \\
\hline Consequences & $.64 *(.11)$ & $.03(.07)$ & $-.46^{*}(.14)$ \\
\hline Self-credit & $.58 *(.06)$ & $.02(.09)$ & $-.03(.10)$ \\
\hline Broadening & $.51 *(.06)$ & $-.13(.11)$ & $-.01(.06)$ \\
\hline Replaying & $.51 *(.06)$ & $-.03(.06)$ & $-.09(.11)$ \\
\hline Capitalizing & $.44 *(.05)$ & $.04(.12)$ & $.12(.10)$ \\
\hline Reminiscing & $.42 *(.07)$ & $-.21 *(.09)$ & $-.05(.08)$ \\
\hline Future Focus & $.39 *(.07)$ & $-.15(.12)$ & $.08(.08)$ \\
\hline Social sharing & $.32 *(.08)$ & $-.09(.10)$ & $.01(.06)$ \\
\hline Other-credit & $.30 *(.06)$ & $-.06(.09)$ & $-.03(.07)$ \\
\hline Stimulus control & $.27 *(.06)$ & $-.04(.10)$ & $.13(.07)$ \\
\hline Expressive suppression & $-.32 *(.13)$ & $.87 *(.08)$ & $-.00(.02)$ \\
\hline Emotional expression & $.02(.05)$ & $.70 *(.07)$ & $.10(.10)$ \\
\hline Minimizing & $-.07(.11)$ & $.61 *(.08)$ & $-.02(.09)$ \\
\hline Denial & $.05(.11)$ & $.57 *(.13)$ & $.21(.13)$ \\
\hline Reappraisal & $.34 *(.10)$ & $.42 *(.10)$ & $-.01(.04)$ \\
\hline Substance use & $-.01(.03)$ & $.38(.21)$ & $.69 *(.10)$ \\
\hline Reward & $.11(.08)$ & $.30(.20)$ & $.54 *(.07)$ \\
\hline Behavioural activation & $.02(.07)$ & $.11(.18)$ & $.52 *(.09)$ \\
\hline Entertainment & $-.01(.06)$ & $.28(.16)$ & $.45 *(.07)$ \\
\hline
\end{tabular}

Between-person

\begin{tabular}{|c|c|c|c|}
\hline Variable & Enhancement & Disengagement & Behavioural \\
\hline Broadening & $.92 *(.08)$ & $-.11(.16)$ & $-.12(.12)$ \\
\hline Replaying & $.90 *(.12)$ & $.03(.12)$ & $-.28 *(.12)$ \\
\hline Savouring & $.87 *(.08)$ & $-.28(.16)$ & $.00(.03)$ \\
\hline Future Focus & $.86 *(.09)$ & $-.09(.18)$ & $-.03(.12)$ \\
\hline Reminiscing & $.80 *(.08)$ & $-.04(.14)$ & $.01(.11)$ \\
\hline Self-credit & $.75 *(.08)$ & $.20(.13)$ & $.07(.12)$ \\
\hline Social sharing & $.74 *(.15)$ & $-.12(.14)$ & $.36 *(.15)$ \\
\hline Consequences & $.69 *(.13)$ & $.38 *(.16)$ & $-.20(.13)$ \\
\hline Capitalizing & $.55 *(.12)$ & $.31 *(.13)$ & $.22(.12)$ \\
\hline Stimulus control & $.46 *(.12)$ & $.23(.14)$ & $.12(.12)$ \\
\hline Other-credit & $.43 *(.13)$ & $.37 *(.15)$ & $-.03(.12)$ \\
\hline Denial & $.00(.03)$ & $.93 *(.08)$ & $-.18(.17)$ \\
\hline Emotional expression & $.08(.10)$ & $.86 *(.08)$ & $-.08(.17)$ \\
\hline Expressive suppression & $-.03(.14)$ & $.84 *(.10)$ & $-.07(.19)$ \\
\hline Minimizing & $.08(.18)$ & $.84 *(.11)$ & $.03(.11)$ \\
\hline Reappraisal & $.41 *(.14)$ & $.65 *(.12)$ & $.02(.05)$ \\
\hline Entertainment & $.49 *(.19)$ & $.01(.04)$ & $.68 *(.10)$ \\
\hline Reward & $.41 *(.20)$ & $.23(.12)$ & $.62 *(.12)$ \\
\hline Substance use & $-.02(.03)$ & $.48 *(.12)$ & $.43 *(.14)$ \\
\hline Behavioural activation & $.45^{*}(.14)$ & $-.01(.09)$ & $.41 *(.12)$ \\
\hline \multicolumn{4}{|l|}{ Correlations } \\
\hline \multicolumn{4}{|l|}{ Enhancement } \\
\hline Disengagement & $.34 *$ & & \\
\hline Behavioural & .13 & .12 & \\
\hline
\end{tabular}

Enhancement

Disengagement 
